\title{
Research on Feature Extraction for Flaw Ultrasonic Echo Based on Wavelet Pack Analysis
}

\author{
Wang Hai-Tao and Zhang Ze
}

\begin{abstract}
Non-destructive testing (NDT) is the technology of using the characteristics of sound, light and magnetic to detect all kinds of physical properties and organization of metal components without any damage to them in order to identify the defects of surface and inner of them. With the development of NDT technology, ultrasonic wave is used to detect the bonding defects of metal thin composite plate by more and more people because of its advantage of greater penetration and higher sensitivity. This paper applies wavelet analysis as the tool of processing signals to extracting features and proposes a new method of filtering based on the traditional wavelet soft and hard threshold filtering algorithm. Because of the limitation of the traditional wavelet theory, this paper applies wavelet pack technology to extracting energy torque of the echo of ultrasonic wave. Decomposed by wavelet pack, the original signal is divided into several frequency bands, whose number is relate to the number of decomposition level. Calculate the energy torque of each frequency band, and the results show that the energy torque can be viewed as the feature used to recognize the bonding defects of the metal thin composite plate.
\end{abstract}

Index Terms-Non-Destructive testing, echo of ultrasonic wave, feature extraction, wavelet transformation, energy torque.

\section{INTRODUCTION}

With the development of modern industry and scientific technology, production equipment is becoming increasingly large, integrated and intelligent; the management for equipment, which is more and more important in production, puts forward higher requirements, so NDT technology is born and attracts more and more researchers, becoming increasingly popular in the field of fault diagnosing for large-scale equipment. Ultrasonic testing is a kind of non-destructive testing. When ultrasonic wave penetrates into the object and attaches the defects, some of ultrasonic wave will be reflected, the reflected wave can be analyzed by receiver, which shows the defects of object precisely. Ultrasonic wave is applied to detecting the bonding defects of metal thin composite thin plate increasingly widely because of its advantage of greater penetration and higher sensitivity [1]-[9].

Wavelet transformation can divide the signal gradually in multi-scale by means of dilation and translation; it will attain time refinement in high-frequency and frequency refinement in low-frequency at length [2]-[3]. It adapts automatically to the requirements of time-frequency signal analysis, so any

Manuscript received November 15, 2012; revised April 16, 2013.

Wang Hai-tao is with the Electrical Information Engineering Department of Inner Mongolia University (e-mail: zhangzeimu@163.com).

Zhang $\mathrm{Ze}$ is with the Automation Department of Inner Mongolia University. details of signal can be focused [4]. Wavelet transformation resolves the problems which Fourier transformation can't resolve, and becomes the second breakthrough in scientific technique after Fourier transformation [4]. Compared with wavelet decomposition, wavelet pack decomposition is a more meticulous algorithm, not only does it decomposes the low-frequency part, but also the high-frequency part, it has the characteristic of higher adaptability [5], so all the features of signal can be remained, it is suitable to extract the features of signal [6]-[8].

In terms of the ultrasonic echo that detects the bonding defects in metal thin composite plate, this paper proposes a new threshold filtering method based on traditional wavelet hard and soft threshold algorithm to filter the echo of ultrasonic wave [2]-[4], applies wavelet pack technology to extracting energy torque of the signal [8]-[9], this method remains the original features in each frequency band of ultrasonic echo wave and has better effect.

\section{PRINCIPLE OF WAVELET TRANSFORMATION}

Wavelet transformation is a kind of time-frequency field partial analysis that both time window and frequency window are variable. It has higher frequency resolution in low-frequency band and higher time resolution in high-frequency band. Because of this characteristic, wavelet transformation has adaptability.

Wavelet function can be defined as follows: Assuming that $\psi(t)$ is square integrable, that is to say, $\psi(t) \in L^{2}(R)$. If its Fourier transformation $\psi(\omega)$ meets the admissible condition:

$$
C_{\psi}=\int_{-\infty}^{+\infty} \frac{|\psi(\omega)|^{2}}{|\omega|} d \omega<\infty
$$

Then $\psi(t)$ is called to be the basic wavelet function or wavelet mother function. $\psi(t)$ is changed to be a wavelet sequence $\psi_{(a, b)}(t)$ by means of dilation and translation [2].

$$
\psi_{(a, b)}(t)=|a|^{-\frac{1}{2}} \psi\left(\frac{x-b}{a}\right)
$$

where, $a$ and $b \in R$ and $a \neq 0$. $a$ is dilation factor and $b$ is translation factor. It can be defined that (2) is the continuous wavelet transformation of the basic wavelet function $\psi(t), X$ is conjugate operation of $X$.

The essence of wavelet transformation is transforming any function $f(t)$ in $L^{2}(R)$ space into the superposition of its projections on $\psi_{a, b}(t)$ whose dilation factor $a$ and translation factor $b$ are variable. Be different from Fourier transformation, wavelet transformation maps 
one-dimensional time-domain signal to the "time - scale", which is two-dimensional domain. So the expand of $f(t)$ in wavelet function has the characteristic of multi-resolution.

In numerical calculation of practical problems, the continuous wavelet transformation should be changed into the discrete one, which is shown as (3).

$$
\left(W_{\psi} f\right)(a, b)=\left\langle f, \psi_{a, b}\right\rangle=\left|a_{0}\right|^{-\frac{m}{2}} \int_{-\infty}^{+\infty} f(t) \overline{\psi\left(a_{0}^{-m} t-n b_{0}\right)} d t
$$

where $a_{0}=a^{1 / m}, b_{0}=b / n a_{0}{ }^{m}$. It can be proved in theory that the basic information of signal will not be lost when the continuous wavelet transformation is changed into the discrete one. Besides, the orthogonality of wavelet function will eliminate the association caused by redundancy between two points in wavelet space.

\section{The FILTERING AlgORITHM BASED ON THE IMPROVED WAVELET THRESHOLD}

Noise always exists in echo of practical ultrasonic wave. In order to extract features of signal, we must eliminate the noise doping in the echo of ultrasonic wave before extracting features.

We must abide the following steps when wavelet transformation is applied to filtering digital signal. Firstly, the signal should be decomposed by wavelet. Secondly, because the noise always exists in higher frequency details, the decomposed wavelet coefficients can be processed by threshold. Finally, reconstruct the signal by means of wavelet transformation. Then the noise will be eliminated.

The traditional processing of filtering based on wavelet transformation adopts the method of soft and hard threshold; these two methods are applied wildly in practical industry [2] But the continuity of wavelet coefficients in hard threshold processing is bad; the reconstructed signal always has some shocking; although the continuity of wavelet coefficients in soft threshold processing is better, the deviation between estimated wavelet coefficient and composed wavelet coefficient of original signal provokes the loss of high-frequency information, which affects the degree of approximation between reconstructed signal and original signal [3].

In terms of the limitation of the traditional wavelet soft and hard threshold filtering methods, this paper proposes an improved filtering method, bonding the soft and hard functions by means of weighting average, which is shown as equation (4).

$$
w_{j, k}^{\prime}= \begin{cases}0 & \left|w_{j, k}\right|<\lambda_{j 1} \\ \left(1-\mu_{j}\right) \cdot w_{j, k}+\mu_{j} \cdot \operatorname{sign}\left(w_{j, k}\right) \cdot\left(\left|w_{j, k}\right|-\lambda_{j 1}\right) & \lambda_{j 2}>\left|w_{j, k}\right| \geq \lambda_{j 1} \\ \left(1-\mu_{j}\right) \cdot w_{j, k}+\mu_{j} \cdot \operatorname{sign}\left(w_{j, k}\right) \cdot \frac{\lambda_{j 2}-\lambda_{j 1}}{\exp \left[\sqrt{\frac{\left|w_{j, k}\right| \lambda_{j 2}}{\left|w_{j, k}\right|+\lambda_{j 2}}}\right]} & \left|w_{j, k}\right| \geq \lambda_{j 2}\end{cases}
$$

where, $w_{j, k}$ is wavelet coefficient of the $j$ th level, $\mu_{j}$ is weighting factor, $\lambda_{j}$ is the threshold of wavelet coefficient in the $j$ th level.

$$
\mu_{j}=\frac{\lambda_{j 1}}{\left|w_{j, k}\right| \cdot \exp \left(\sqrt{\frac{\left|w_{j, k}\right|-\lambda_{j 1}}{\left|w_{j, k}\right|+\lambda_{j 1}}}\right)}
$$

If $\left|w_{j, k}\right|=\lambda_{j l}, w_{j, k}=0$. When $\left|w_{j . k}\right| \rightarrow \lambda_{j l}, \mu \rightarrow 1, w_{j, k} \rightarrow 0$. If $\left|w_{j . k}\right|=\lambda_{j 2}, w_{j, k}{ }^{\prime}=\left(1-\mu_{j}\right) w_{j, k}+\mu_{j} \operatorname{sign}\left(w_{j, k}\right)\left(\lambda_{j 2}-\lambda_{j 1}\right)$. When $\left|w_{j . k}\right| \rightarrow \lambda_{j 2}$, $w_{j, k}{ }^{\prime} \rightarrow\left(1-\mu_{j}\right) w_{j, k}+\mu_{j} \operatorname{sign}\left(w_{j, k}\right)\left(\lambda_{j z}-\lambda_{j 1}\right)$. So $w_{j, k}$ ' is continuous in the area of $\left|w_{j, k}\right|=\lambda_{j 1}$ and $\left|w_{j, k}\right|=\lambda_{j 2}$. That is to say, this method overcomes the shortage of wavelet hard threshold algorithm. With the increasing of $\left|w_{j, k}\right|$, the variance between $w_{j, k}$ and $w_{j, k}$ ' decreases, when $\left|w_{j, k}\right| \rightarrow \infty, \mu \rightarrow 0, w_{j, k}$ ' $\rightarrow w_{j, k}$, so it also overcomes shortage of wavelet hard threshold algorithm, reducing the variance between $w_{j, k}$ and $w_{j, k}$ '.

Considering the echo of ultrasonic wave is not stable, so an auto-adaptively strategy is proposed in this paper, we set two thresholds $\lambda_{j 1}$ and $\lambda_{j 2}$, the $\lambda_{j}$ is related to the number of level and the wavelet coefficient of each level [2].

$$
\begin{aligned}
& \lambda_{j 1}=\sigma \frac{\sqrt{2 \cdot \ln N_{j}}}{\ln (j+1)} \\
& \lambda_{j 2}=\sigma \sqrt{2 \cdot \ln N} \\
& \sigma=\frac{\operatorname{median}\left(\left|w_{1}\right|\right)}{0.6475}
\end{aligned}
$$

where $N_{j}$ is the length of the $j$ th level wavelet coefficient matrix, $N$ is length of original signal. The number of decomposition level is chosen to be 5 . The function of wavelet is chosen to be 'db4'. $\sigma_{j}$ is the estimated variance of noise, it is estimated by detail signal of the first level. The numerator is the median of these five wavelet coefficients [4].

Take the example of the metal thin composite plate that without any defects in it. The length of sampling is 1000 -point, sampling frequency is $100 \mathrm{MHz}$, and the central frequency of ultrasonic probe is $5 \mathrm{MHz}$. The echo of ultrasonic wave is shown as Fig. 1. Fig. 2, Fig. 3 and Fig. 4 show the results of filtering using traditional wavelet hard threshold, traditional wavelet hard threshold and the improved wavelet threshold, respectively.

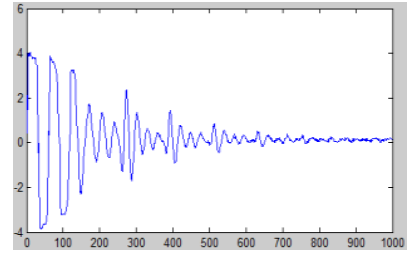

Fig. 1. The original signal.

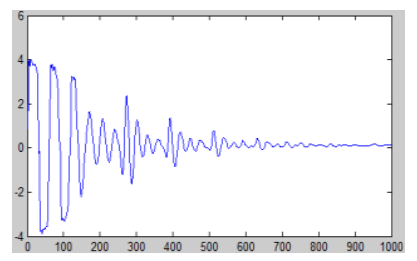

Fig. 3. Filtered signal (soft threshold)

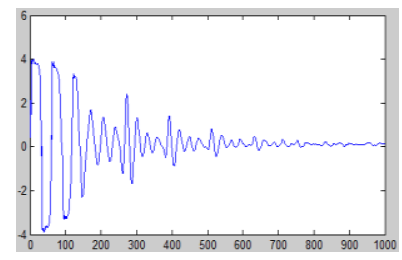

Fig. 2. Filtered signal (hard threshold)

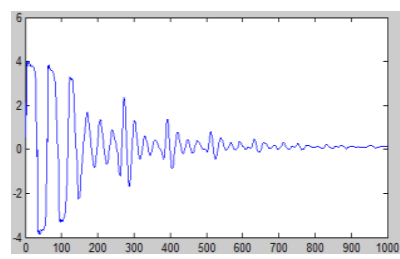

Fig. 4. Filtered signal (improved threshold) 
It is difficult to identify the difference among these three algorithms, whose effect can be illustrated by means of calculating SNR, which is shown in Table I.

TABLE I: SNR OF THESE THREE ALGORITHMS

\begin{tabular}{cccc}
\hline \hline Method of & Wavelet Hard & Wavelet Soft & Improved \\
Filtering & Threshold & Threshold & Wavelet \\
& Filtering & Filtering & $\begin{array}{c}\text { Threshold } \\
\text { Filtering }\end{array}$ \\
& & & 30.4583 \\
\hline SNR & 30.0500 & 25.8710 & \\
\end{tabular}

It can be seen from the Table I, the SNR of filtering algorithm based on wavelet soft threshold is the lowest. The SNR of filtering algorithm based on the improved algorithm is the highest, and its reconstructed signal is smooth enough, it refrains the phenomenon of Gibbs shocking. In summary, the improved threshold algorithm is better in the filtering effect.

\section{FEATURE EXTRACTION}

Features can be extracted from the echo of ultrasonic wave after filtering; the feature extracted form signal must be sensitive to bonding defect [5]. This paper applies wavelet pack decomposition to the echo of ultrasonic wave and extracts the characteristic of energy torque. It can be proved from the results: energy torque of the echo can be used to judge the degree of de-bonding of metal thin composite plate.

\section{A. Principle of Wavelet Pack}

Wavelet packet transformation is a further development of wavelet transformation. It provides higher resolution than that provided by wavelet transformation. Not only does it decomposes the low-frequency part of signal, but also the high-frequency part, it can choose the corresponding frequency-band auto-adaptively according to features of the analyzed signal, making it match the spectrum of signal [5]. Wavelet pack transformation increases the resolution of time-frequency, so its value of application is more expansive [4].

In multi-resolution analysis, $L^{2}(R)=\oplus W_{j}(j \in Z)$, which is to say that multi-resolution analysis divides Hilbert space $L^{2}(R)$ into the orthogonal sum of all subspaces $W_{j}$ according to scale factor $j . W_{j}$ is a closed pack. Now we hope to divide wavelet subspace $W_{j}$ in frequency according to binary fraction in order to improve the resolution of frequency [5].

One of reasonable approaches is to represent scale space $V_{j}$ and wavelet space $W_{j}$ to be a new subspace $U_{j}^{n}$ uniformly [2]. If orthogonal composition of Hilbert space $V_{j+1}=V_{j} \oplus W_{j}$ can be unified to be (10) by the composition of $U_{j}^{n}$.

$$
\begin{gathered}
\left\{\begin{array}{l}
U_{j}^{0}=V_{j} \\
U_{j}^{1}=W_{j}
\end{array} \quad j \in Z\right. \\
U_{j+1}^{0}=U_{j}^{0} \oplus U_{j}^{1} \quad j \in Z
\end{gathered}
$$

Define subspace $U_{j}^{n}$ to be the closed space of function $u_{n}(t)$, $U_{j}^{2 n}$ is the closed space of function $u_{2 n}(t), u_{n}(t)$ meets the double-scale function shown as (11).

$$
\left\{\begin{array}{l}
u_{2 n}(t)=\sqrt{2} \sum_{k \in Z} h(k) u_{n}(2 t-k) \\
u_{2 n+1}(t)=\sqrt{2} \sum_{k \in Z} g(k) u_{n}(2 t-k)
\end{array}\right.
$$

where $g(k)=(-1)^{k} h(1-k)$, these two coefficients are orthogonal with each other. When $n=0$, we will get (12) from (10) and (11) [2]

$$
\left\{\begin{array}{l}
u_{0}(t)=\sum_{k \in Z} h_{k} u_{n}(2 t-k) \\
u_{1}(t)=\sum_{k \in Z} g_{k} u_{n}(2 t-k)
\end{array}\right.
$$

In multi-resolution analysis, $\phi(t)$ and $\psi(t)$ meet double-scale (13).

$$
\begin{cases}\phi(t)=\sum_{k \in Z} h_{k} \phi(2 t-k) & \left\{h_{k}\right\}_{k \in Z} \in l^{2} \\ \psi(t)=\sum_{k \in Z} g_{k} \phi(2 t-k) & \left\{g_{k}\right\}_{k \in Z} \in l^{2}\end{cases}
$$

Compared with (12), $u_{0}(t)$ and $u_{l}(t)$ degenerate to be scale function $\phi(t)$ and wavelet function $\psi(t)$, respectively. Equation (12) is the equivalent representation of Equation (10). Promote this equivalent representation into the condition that $n \in \mathbf{Z}_{+}$, the equivalent representation of (11) will be available, which is shown as follow.

$$
U_{j+1}^{n}=U_{j}^{n} \oplus U_{j}^{2 n+1} \quad j \in Z
$$

Wavelet pack transformation decomposes the low-frequency of signal as well as the high-frequency of signal; the degree of decomposition of high-frequency component is same as that of high-frequency component. In terms of the digital signal $x_{n}, n=1,2, \cdots, N$, there are $2^{i}$ wavelet packs in the $i$ th level of wavelet pack transformation. The length of each pack is $2^{J-j}, J=\log _{2} N$ [2].

The original signal is composed into several wavelet pack coefficients by wavelet pack transformation; each coefficient is a sequence, which correspond a frequency component [5]. Because of the orthogonality of wavelet pack decomposition, wavelet packs in the fixed scale are translation orthogonal with each other, and wavelet packs in different scales have the characteristic of orthogonality. Therefore, we can reconstruct the original signal by means of wavelet packs in the fixed scale or orthogonal basis in different scales.

\section{B. Selection of Level of Wavelet Pack Decomposition and Function of Wavelet Pack}

When wavelet pack is applied to extracting the features of the signal, the appropriate number of decomposition level and the function of wavelet pack must be determined. The largest number of decomposition level is $\log _{2} N$ in theory. In practice, if the number of level is too small, the low-frequency and high-frequency of signal will be overlapping in the same frequency band, so it will be meaningless to extract the energy torque of the whole frequency band. If the number of level is too big, there will be certain of distortion, which is not helpful to reconstruct the 
original signal [6]. Therefore, we must choose the appropriate number of level. In this paper, the number of level is chosen to be 6 .

It is difficult to summarize general rules for the selection of wavelet pack function; generally speaking, wavelet pack functions $\mathrm{db} N$ and $\operatorname{sym} N$ are advantageous over other functions, which are prominent in one or two aspects. In practice, in order to mediate the contradictions among the properties of wavelet pack functions, a compromise must be chosen according to actual requirements [7]. This paper adopts db4 wavelet pack to decompose the echo of ultrasonic wave; and extracts the energy torque of each frequency band.

\section{Extracting Energy Torque Based on Wavelet Pack Decomposition}

The echo of ultrasonic wave that detects the metal thin plate contains a lot of information about the bonding defects of metal thin composite plate [8]. The key of recognizing defects is how to extract the information that reflects the characteristics of defects out of the echo of ultrasonic wave and to give the accurate explanations. Because the ultrasonic wave has characteristic of non-stability, the method of extraction is especially important [8]. Signal can be decomposed to be many components in frequency band with any degree of precision [9], then we calculate energy torques of decomposed signals in each frequency band and assemble them to be an eigenvector, the results show that this method has better effect.

It is known that the energy of signal is related to wavelet coefficient from Parseval equation that is shown as (15).

$$
\int_{-\infty}^{+\infty}|f(x)|^{2} d x=\sum_{k}\left|w_{j, k}\right|^{2}
$$

That is to say, wavelet coefficient has the gauge of energy of signal, so it can be applied to analysing the energy of signal. Based on this conclusion, this paper proposes the method of extracting energy torque of each frequency band to distinguish the different bonding defects of metal thin composite plate [9].

Assuming that $S$ is the filtered signal, $X_{i j}$ is wavelet pack coefficient of the $j$ th node in the $i$ th level, $S_{i j}$ is wavelet pack coefficient of the reconstruction for $X_{i j}$.

$$
S=\sum_{j=1}^{2^{i}} S_{i j}
$$

where $i$ is the number of wavelet pack composition level, $S$ is composed to be $2^{i}$ frequency bands, $E_{i j}$ is the energy of $S_{i j}$, $x_{i j}$ is amplitude of $S_{i j}, n$ is the length of $S_{i j}$.

$$
E_{i j}=\int\left|S_{i j}(t)\right|^{2} d t=\sum_{j=1}^{n}\left|x_{i j}\right|^{2}
$$

$E_{i j}$ shows energy of the whole frequency, without considering the time-varying of frequency, the echo is non-stable, so its frequency changes with time. So besides the distribution of energy in the whole frequency, we must consider the distribution of energy with time. We can introduce the time lever; so energy is changed to be energy torque, as it is shown in (18).

$$
E_{i j}^{\prime}=\int\left|S_{i j}(t)\right|^{2} d t=\sum_{j=1}^{n}\left|x_{i j}\right|^{2} \cdot N_{i j}
$$

where $N_{i j}$ is the length of wavelet pack coefficient of the $j$ th node in the $i$ th level.

This paper applies this method to detecting three bonding defects, $0 \%, 40 \%$ and $80 \%$ de-bonding, the filtered signal is decomposed by wavelet pack transformation, the number of decomposition level is 6 , the signal is decomposed to be 64 frequency bands averagely, the energy torque of each frequency is shown as follows.

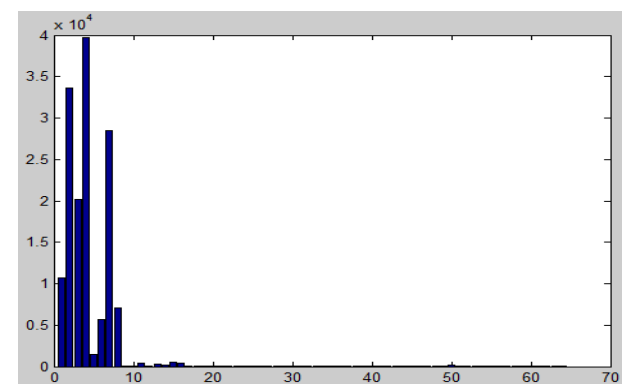

Fig. 5. Energy torque of echo detecting plate without bonding defects.

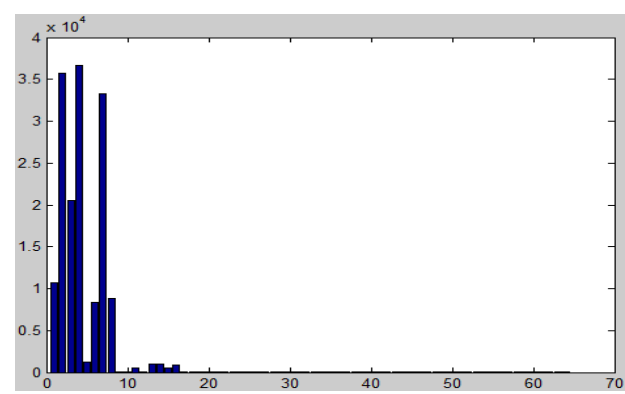

Fig. 6. Energy torque of echo detecting plate with $40 \%$ bonding defects.

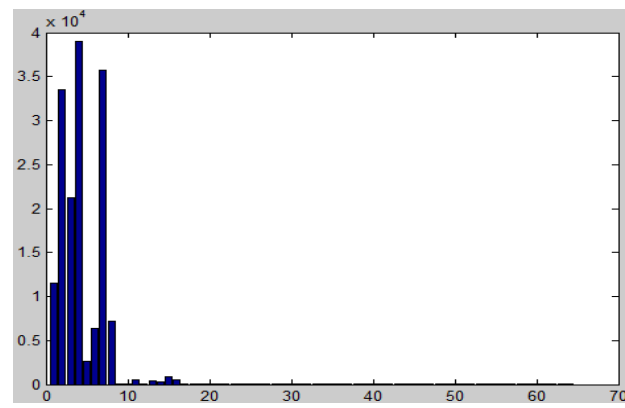

Fig. 7. Energy torque of echo detecting plate with $80 \%$ bonding defects.

The frequency of echo varies from $0 \mathrm{~Hz}$ to $8 \mathrm{MHz}$, so when the echo is composed by wavelet pack, the first frequency band is from $0 \mathrm{~Hz}$ to $0.125 \mathrm{MHz}$, the second one is form $0.125 \mathrm{MHz}$ to $0.25 \mathrm{MHz}$, the rest may be deduced by analogy [9].

Through these three figures, it can be seen that the energy torque of these three signals are different from each other. Energy torque concentrates in the first eight frequency bands, the variance among energy torques of the fourth and seventh frequency band is larger, which is to say, bonding defects affect energy torque of the fourth and seventh frequency band, so we can infer that the defects reflects the signal 
components of the fourth and seventh frequency, which vary from $0.375 \mathrm{MHz}$ to $0.5 \mathrm{MHz}$ and from $0.75 \mathrm{MHz}$ to $0.875 \mathrm{MHz}$, respectively. If energy torque of the $i$ th frequency band is represented to be $E_{i}$, energy torques in the $4^{\text {th }}$ and $7^{\text {th }}$ frequency band of the echo is shown in Table II. The difference reflects the difference among these three signals in order to judge the degree of de-bonding.

TABLE II: COMPARISON OF ENERGY TORQUES

\begin{tabular}{ccc}
\hline \hline Energy torque & $E_{4}$ & $E_{7}$ \\
\hline $0 \%$ de-bonding & $3.9653 \times 10^{4}$ & $2.8513 \times 10^{4}$ \\
$40 \%$ de-bonding & $3.6703 \times 10^{4}$ & $3.3293 \times 10^{4}$ \\
$80 \%$ de-bonding & $3.8987 \times 10^{4}$ & $3.5710 \times 10^{4}$ \\
\hline \hline
\end{tabular}

As all we know, the defects will reflect the ultrasonic wave, but not absorb it. When ultrasonic wave attaches the bonding defects, the energy of echo will increase. So the degree of difference among energy torques of each frequency band will be larger, the larger the degree of de-bonding is, the larger the difference is. We can calculate the variance of these three pairs of energy torques, which are shown in Table III.

TABLE III: COMPARISON OF VARIANCE OF ENERGY TORQUES

\begin{tabular}{cccc}
\hline \hline $\begin{array}{c}\text { Degree of } \\
\text { de-bonding }\end{array}$ & $\begin{array}{c}0 \% \\
\text { de-bonging }\end{array}$ & $\begin{array}{c}40 \% \\
\text { de-bonging }\end{array}$ & $\begin{array}{c}80 \% \\
\text { De-bonging }\end{array}$ \\
\hline $\mathrm{D}$ & $5.9904 \times 10^{7}$ & $6.3800 \times 10^{7}$ & $6.6640 \times 10^{7}$ \\
\hline \hline
\end{tabular}

It can be seen from Table III that the variance increases with the degree of de-bonging, it can be viewed as one of features to recognize the degree of de-bonding. So the components $E_{4}, E_{7}$ and $D$ can be assembled to be an eigenvector to distinguish the degree of de-bonding.

\section{CONCLUSIONS}

Wavelet packet analysis has become one of the fastest growing and the most attractive subjects in areas of signal processing, and it is gradually applied to signal. This paper applies wavelet analysis to filtering the echo of ultrasonic wave which detects the bonding defects successfully, the results show that wavelet analysis can filter signal in different ranges of frequency. Meanwhile, wavelet pack analysis is applied to extracting the features of the filtered signal; it is found that energy torque and the variance of it can be viewed as the features of echo to identify the different echo waves. Through the comparison among the energy torques, the bonding defects of metal thin composite plate can be distinguished effectively.

\section{ACKNOWLEDGMENT}

This work is supported by Nature Science Fund of China Grant \# 60862004 to Zhang Ze.

\section{REFERENCES}

[1] Y. Sano and T. Yoshino, "Fast optical wavelength interrogator employing arrayed waveguide grating for distributed fiber-bragg-grating sensors," Journal of Light wave Technology, 2003, vol. 21, no. 1, pp. 132139.

[2] H. Y. Zhang, Q. Zhou, and J. D. Xia, "Wavelet packet de-noising and feature extraction for flaw echo signal in ultrasonic testing," Chinese Journal of Scientific Instrument, vol. 127, no. 1, 2006.

[3] S. Z. Zeng, The algorithm of wavelet de-noising and its application in signal processing.

[4] G. M. Shi, "The de-noising method of the drill testing signal based on wavelet transform," Science and Technology Information 2010, vol. 19.

[5] D. Y. Jiang, "The study of ECG feature extraction method based on wavelet (packet) transformation," Dissertation, Southwesten University, 2009.

[6] H. Y. Zhang, "Application of wavelet analysis and fuzzy pattern recognition to flaw identification in ultrasonic testing," Nondestructive Testing, vol. 22, no. 2, pp. 51-54, 2000.

[7] M. V. Wickerhauser, "Adapted wavelet analysis from theory to software," The Institute of Electrical and Electronics Engineers, IEEE, Inc. New York, 1994.

[8] J. Y. Zhang, B. Zhang, and X. Z. Jiang, "The method of feature extraction based on wavelet transformation," Signal Processing, vol. 16, no. 2, 2000.

[9] Z. Zhang and Y. Q. Ren, "Time-Frequency analysis of echoes signal in ultrasonic testing of adhesion based on short-time fourier transformation," in Proc. 2010 International Conference on Measuring Technology and Mechatronics Automation, IEEE Computer Society, pp. 1023-1026, Mar. 2010.

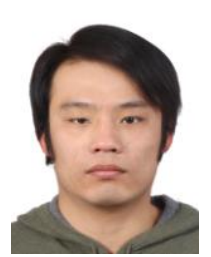

Wang Hai-Tao was born in TianJin in Sep, 1983. He graduated from automation department of Inner Mongolia University in Jul, 2006 and received the bachelor degree in specialized subject of automation. From Jul, 2006 to Jul, 2009, he worked for SINOPEC and assigned to the project of natural gas expansion in Saudi Arab. Then he returned to Inner Mongolia University and strived to mater degree, and his specialized subject is pattern recognition and intelligent system. In Jul, 2012, he graduated from electrical information engineering department of Inner Mongolia University and got master degree.

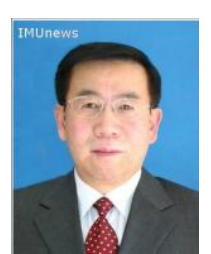

Zhang Ze was born in Hohhot, Inner Mongolia in Feb, 1951. He graduated from physics department of Inner Mongolia University in Jul, 1977 and received the bachelor degree in specialized subject of radio. And then he learned master courses form Aug, 1984 to Jul, 1985 in radio department of Tsinghua University. Zhang Ze taught in electrical engineering department of Inner Mongolia University from May, 1979 to Oct, 1999 and worked as an assistant, lecturer and vice professor in succession. He strived to the field of circuit design and signals processing and was ascended to be master instructor in field of signal and information processing. From Oct, 1999 to May, 2007, he worked as a professor and dean in automation department of Inner Mongolia University, striving to research on signal processing, pattern recognition and automatic control, and then he worked as a master instructor in pattern recognition and intelligent system. Now, he works as a professor in automation of Electrical Information Engineering College of Inner Mongolia University. Zhang Ze has been a member of the guiding branch-committee of electrical information science and engineering since 2006, by far from 2008, he has been a member of signal processing expert committee of Seminar of Chinese high-tech industrialization. 